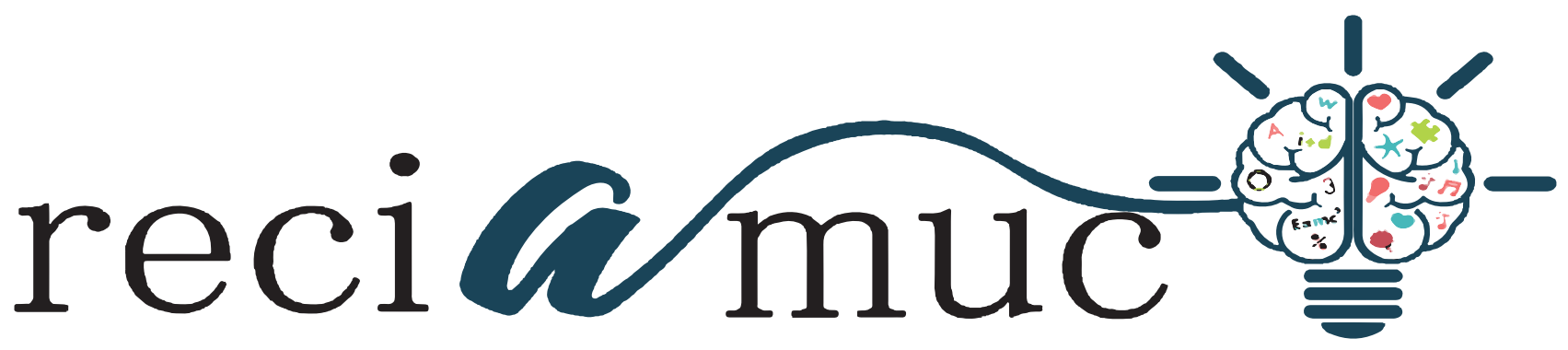

DOI: 10.26820/reciamuc/5.(4).noviembre.2021.22-36

URL: https://reciamuc.com/index.php/RECIAMUC/article/view/718

EDITORIAL: Saberes del Conocimiento

REVISTA: RECIAMUC

ISSN: 2588-0748

TIPO DE INVESTIGACIÓN: Artículo de Revisión

CÓdigo UNESCO: 33 Ciencias Tecnológicas

PAGINAS: 22-36

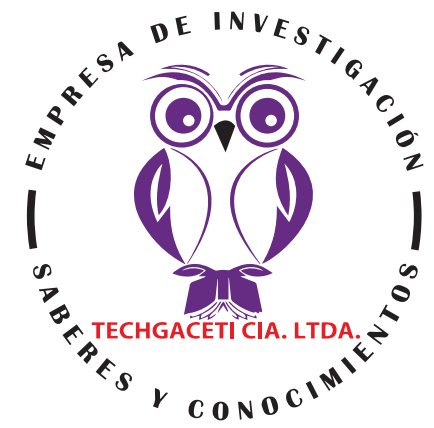

\title{
TIC y su contribución para el desarrollo sostenible en la agroindustria alimentaria
}

ICT and its contribution to sustainable development in the food industry TIC e sua contribuição para o desenvolvimento sustentável na indústria de alimentos

\section{Jorge Enrique Ordoñez García'; Christian Elías Merizalde Andrade2; Walter Giovanny Villamar Piguave ${ }^{3}$}

\section{RECIBIDO: 15/09/2021 ACEPTADO: 05/10/2021 PUBLICADO: 29/11/2021}

1. Master Universitario en Didáctica de las Matemáticas en Educación Secundaria y Bachillerato; Magister en Automatización y Control Industrial; Ingeniero en Electrónica y Telecomunicaciones; Docente de la Universidad de Guayaquil, Ecuador; jorge.ordonezga@ug.edu.ec; (iD https://orcid.org/0000-0002-0888-7481

2. Magister en Sistemas de Información mención en Inteligencia de Negocios; Ingeniero en Sistemas con mención en Telemática; Docente de la Universidad de Guayaquil; Guayaquil, Ecuador; christian.merizalde@ug.edu. ec; (iD https://orcid.org/0000-0002-0982-6752

3. Magister en Administración de Empresas mención Recursos Humanos y Marketing; Contador Público Autorizado; Profesor de Segunda Enseñanza Especialización Informática; Licenciado en Ciencias de la Educación mención informática; Docente de la Universidad de Guayaquil, Ecuador; walter.villamarpi@ug.edu.ec; (iD https://orcid.org/0000-0002-6622-9460

\section{CORRESPONDENCIA \\ Jorge Enrique Ordoñez García \\ jorge.ordonezga@ug.edu.ec \\ Guayaquil, Ecuador}

() RECIAMUC; Editorial Saberes del Conocimiento, 2021 


\title{
RESUMEN
}

El incremento en la productividad en diversas empresas alrededor del mundo se debe en gran medida al aprovechamiento en el uso de las Tecnologías de la Información y la Comunicación (TIC); ya que permiten la interacción de grandes volúmenes de datos de información a escala planetaria en tiempo real. A partir de la pandemia por COVID-19, se ha incrementado la importancia del uso de la TIC en los procesos innovación de productos, gestión, logística y control. El desarrollo de la economía digital modifica los modelos de gestión, reduce los costos de transacción e intermediación, y explota la información proveniente de los datos que se intercambian en las plataformas digitales, optimizando los procesos. La inteligencia artificial, robótica, realidad aumentada, posibilita la automatización y mayor eficiencia en toda la cadena de valor. La presente investigación documental ahonda en cómo las TICS contribuyen en la industria agroalimentaria, desde la adquisición de insumos: compras, cultivo, recolección, transporte, transformación, hasta el eslabón de la distribución al cliente final. Entre las limitaciones de la digitalización económica, destaca el acceso y el desplazamiento de la mano de obra, ante lo cual la gobernanza y empresas deben avocar esfuerzo e inversión en sus plataforma digitales enmarcado en el desarrollo sostenible, es decir, crecimiento económico basado en generación de empleo, reducción de desigualdad y mayor sostenibilidad; factores clave para estar en la vanguardia de los desafíos que representa para el sector agroalimentario la digitalización como alternativa para producir con menor impacto en el Cambio climático.

Palabras clave: Tecnología, Economía, Agroalimentario, Desarrollo Sostenible, TIC.

\begin{abstract}
The increase in productivity in various companies around the world is largely due to the use of Information and Communication Technologies (ICT); since they allow the interaction of large volumes of information data on a planetary scale in real time. Since the COVID-19 pandemic, the importance of the use of ICT in product innovation, management, logistics and control processes has increased. The development of the digital economy modifies management models, reduces transaction and intermediation costs, and exploits information from data exchanged on digital platforms, optimizing processes. Artificial intelligence, robotics, augmented reality, enable automation and greater efficiency throughout the value chain. This documentary research delves into how ICTs contribute to the agri-food industry, from the acquisition of inputs: purchases, cultivation, collection, transportation, transformation, to the link of distribution to the end customer. Among the limitations of economic digitization, the access and displacement of the workforce stands out, in view of which the governance and companies must commit effort and investment in their digital platforms framed in sustainable development, that is, economic growth based on generation of employment, reduction of inequality and greater sustainability; key factors to be at the forefront of the challenges that digitization represents for the agri-food sector as an alternative to produce with less impact on climate change.
\end{abstract}

Keywords: Technology, Economy, Agri-food, Sustainable Development, ICT.

\section{RESUMO}

O aumento da produtividade em várias empresas ao redor do mundo se deve em grande parte ao uso das Tecnologias da Informação e Comunicação (TIC); uma vez que permitem a interação de grandes volumes de dados de informação em escala planetária em tempo real. Desde a pandemia COVID-19, a importância do uso das TIC na inovação de produtos, gestão, logística e processos de controle aumentou. O desenvolvimento da economia digital modifica os modelos de gestão, reduz os custos de transação e intermediação e explora as informações dos dados trocados em plataformas digitais, otimizando processos. Inteligência artificial, robótica, realidade aumentada permitem automação e maior eficiência em toda a cadeia de valor. Esta pesquisa documental investiga como as TICs contribuem para a indústria agroalimentar, desde a aquisição de insumos: compra, cultivo, coleta, transporte, transformação, até o elo de distribuição ao cliente final. Dentre as limitações da digitalização econômica, destaca-se o acesso e deslocamento da força de trabalho, em vista do qual a governança e as empresas devem empenhar esforços e investimentos em suas plataformas digitais enquadradas no desenvolvimento sustentável, ou seja, o crescimento econômico baseado na geração de empregos, redução da desigualdade e maior sustentabilidade; fatores-chave para estar na vanguarda dos desafios que a digitalização representa para o setor agroalimentar como alternativa para produzir com menor impacto nas mudanças climáticas.

Palavras-chave: Tecnologia, Economia, Agroalimentar, Desenvolvimento Sustentável, TIC. 


\section{Introducción}

Las Tecnologías de la Información y la Comunicación (TIC) han tenido un papel protagónico en los últimos años por el avance tecnológico que se ha desarrollado a partir del siglo XXI, logrando que muchos de los procesos habitualmente realizados por el ser humano puedan ser ejecutados por máquinas, robots o dispositivos. Plantear un concepto preciso para definir las TIC, resulta complejo debido a la amplitud, diversidad y continuo desarrollo que ha alcanzado; sin embargo, se puede referir lo esbozado por el (Instituto Nacional de Estadística y Censos del Ecuador, 2015):

"Las Tecnologías de la Información y la Comunicación (TIC) han transformado la manera de trabajar y gestionar recursos en el ámbito empresarial y se refieren al conjunto de avances tecnológicos que nos proporcionan la informática, las telecomunicaciones y las tecnologías audiovisuales, que comprenden los desarrollos relacionados con los ordenadores, internet, la telefonía, los medios de comunicación de masa, las aplicaciones multimedia y la realidad virtual"

En efecto, las TIC han sido un desafío importante para el desarrollo de las empresas, ya que las mismas se han potenciado a través de la globalización, convirtiéndose en una ventaja competitiva, incluso con el riesgos de dejar fuera a las empresas que no se adapten a esta nueva realidad.

Actualmente se pueden referir múltiples casos de sectores que han volcado sus modos de producción hacia la digitalización plena; así CEPAL, (2021) destaca el uso de la tics en ciertos casos del sector de la hostelería, "donde existen hoteles sin personal que, mediante máquinas y robots, consiguen que el alojamiento funcione sin la necesidad de que humanos interaccionen con sus clientes"
De tal manera que el sector agroalimentario no se ha quedado atrás, de hecho existen establecimientos de alimentación, tales como restaurantes, supermercados, y otros que se han orientado en este misma vía, en donde el cliente puede solicitar su pedido e incluso pagar de forma automática. . "Existen algunos casos más avanzadas donde van mucho más lejos con restaurantes totalmente automatizados donde no hay camareros ni cajeros como es el caso de Estas en Estados Unidos que cuenta ya con 4 restaurantes" (Camara Valencia, 2021)

Estas herramientas a lo interno de las empresas permiten la mejora de sus operaciones y la optimización de sus recursos, a través del procesamiento de un gran número de información, organizándola para presentarla de una forma más sencilla y oportuna. De esta forma, los directivos pueden obtener un mejor conocimiento del desarrollo de cada una de sus operaciones, y tomar decisiones más acertadas. Sin embargo, la accesibilidad de las TIC para todos, aún es utopía, ya que existe una amplia brecha de conectividad entre las zonas urbanas y rurales (ITU, 2021). No obstante; las tics no han de ser vistas como amenaza para las organizaciones de cualquier sector económico, por el contrario representan una potencial de desarrollo tanto para sus procesos y su rendimiento económico, así como para garantizar los lineamientos necesarios propios del desarrollo sostenible requeridos ante el actual cambio climático que se vive a escala planetarias

Así por ejemplo en el caso de agroalimentario, señala a Domene, (2019) que existe una potencialidad en el sistema de trazabilidad, el cual por ejemplo, de acuerdo Carrefour España innovaría lanzando un sistema de trazabilidad alimentaria blockchain, tecnología de almacenamiento y transmisión de información que le permitiría " seguir el rastro de un bien en todas las etapas de producción, transformación y distribución, alcanzando los máximos niveles de seguridad" (Domene, 2019) 
La propagación de la pandemia a escala mundial, ha permitido no solo el desarrollo tecnológico en las fuerzas productivas, sino que de igual forma el usuario final y la sociedad actual, es cada vez más demandante entorno a los datos de la información del alimento que consumirá, así el cliente hoy en día requiere estar en conocimiento de todo el proceso de cultivo, cría y fabricación sobre el alimento que consumirá; así como la minimización del tiempo de la adquisición del producto, exigen los tiempos de hoy sea más eficaz, "una cadena de distribución más corta e. integrada en los procesos de los clientes, puede lograrse con herramientas que digitalicen cada uno de los procesos" (Domene, 2019)

Por lo tanto, el presente artículo analiza las aplicaciones de las TICs en el sector agroalimentario, y su potencial uso. Advierte la importancia de la sofisticación del proceso productivo agroalimentario como un elemento vital para subsistir en el mundo de hoy, existe basta documentación que será analizada para referir las herramientas tecnológicas aplicada en la actualidad en la digitalización de la economía, para referir su contribución en los procesos agro alimentario en el marco del desarrollo sostenible que exige la sociedad ante el cambio climático.

\section{Metodología}

El presente artículo expone las contribuciones de las TIC en la industria agroalimentaria, bajo un enfoque descriptivo resultante de una investigación minuciosa de diferentes literaturas. El criterio de selección de las fuentes seleccionadas consistió en el año de publicación superior a abril de 2020, ya que la economía ha dado un giro importante con nuevos enfoques en su modelo de gestión y en la cadena de producción, a causa del vertiginosidad que han desarrollado las tecnología de información y comunicación que se vieron obligadas a dar un salto cuali cuantitativo, a partir del cierre de los establecimientos de la mayoría de los países del mundo debido a la pandemia COVID 19.
En consecuencia, las bibliografías utilizadas anteriores a esta fecha, sirven de elemento referencial comparativo, y para concepciones teóricas respecto a la agroindustria y aplicación de las TICs. Así se analizaron las variables: TIC, Economía digital, Tendencia de adquisición de dispositivos tecnológicos de las empresas, Tics y sus efectos en las cadenas de producción en la agroindustria,

Toda la información fue recabada, ordenada y clasificada, desestimando las de menor vinculación a las variables principales. Por último, se analizó toda la data, se sintetizó y se organizó para presentarla bajo un orden correlativo temporal

\section{Resultados}

\section{Tecnologías de la información y la comu- nicación}

La idea conceptual del término TIC refiere en esencia a las Tecnologías para comunicar e informar, por lo cual se puede definir como "las tecnologías que utilizan la informática, la microelectrónica y las telecomunicaciones para crear nuevas formas de comunicación a través de herramientas de carácter tecnológico y comunicacional (ordenadores, teléfonos, televisores, entre otros), con la finalidad de facilitar la emisión, acceso y tratamiento de la información" (Hernández, 2021). El término TIC, (en inglés ICT: Information and Communications Technology), se origina a principio de los 90 para sustituir a el término N'TIC, (Nuevas Tecnologías de Información y Comunicación) utilizado durante el inicio de este siglo (Calandra \& Araya , 2009).

Dado que el crecimiento tecnológico acelerado durante el siglo XXI y con mayor ahínco luego del surgimiento de la pandemia COVID 19, las TIC actualmente reflejan la innovación y renovación tecnológica años tras año, combinando la radio, la telefonía y la televisión con la digitalización de las tecnologías de registro de datos, y con el desarrollo de redes, y de la Internet. De esta manera, las personas puedan comunicarse sin 
importar la distancia, oír o ver situaciones que ocurren en otro lugar y, las más recientes, poder trabajar o realizar actividades de forma virtual, a través del correo electrónico, la búsqueda y el filtro de la información, descarga de materiales, comercio en línea, e-learning, Apps, etc (Monroy, 2020).

En tal sentido, el acceso a la información y la capacidad para transformarla permite el la eficiencia y la transformación en el ámbito económico, así las empresas y organizaciones de todo ámbito, el nivel de vida de las sociedades de todo el mundo ha mejorado considerablemente en su mayoría, por lo cual es tenida en cuenta como indicador importante en los estudios de desarrollo económico (Hernández, 2021).

En efecto las tics permiten el acercamiento con sus clientes, rompiendo barreras, distancias y conquistando nuevos mercados (Espinal, 2019):

"Las organizaciones se han dado cuenta que el empleo de las nuevas tecnologías permiten, en primer lugar, llegar más rápido al mercado y cubrir un mercado más amplio, ya que las distancias no son un límite; además, brindan la oportunidad de dar una imagen de empresa innovadora frente a la competencia".

En efecto el hecho de disponer en tiempo real de la información para la toma de decisiones a lo largo de toda una cadena de valor es una ventaja competitiva que permite a las empresas usar eficientemente de los recursos y dar respuesta oportuna a la demanda, con soluciones basadas en la "nube" posibilita la integración expedita de los procesos productivos a lo largo de la cadena de valor. Asi en el caso de las operaciones, se puede señalar como con una misma aplicación y/ o software puede ser desarrollada para diseñar, simular y desplegar de las instrucciones para ejecutar líneas de producción físicas, lo que permite flexibilizar y mejorar el rendimiento operacional; lo que remplaza al modo de producción tradicional, optimizándolo y disminuyendo costos asociados, el tiempo de entrega mejorar la gestión logística y captar la atención de los consumidores. (CEPAL, 2021)

Se puede señalar de igual manera, la importancia de otros usos con sistemas de automatización y control industrial, de planificación y diseño, y los dispositivos de campo que entregan información para la optimización completa del proceso. De igual forma, la incorporación de la inteligencia artificial en los procesos de decisión permite optimizar la gestión de recursos hacia una menor huella ambiental en ámbitos como la explotación de recursos naturales, la manufactura, la logística y el transporte, y el consumo.

A continuación se presenta grafico que indica la contribución que proporciona las TICS a las cadenas de producción de distintos sectores económicos: 


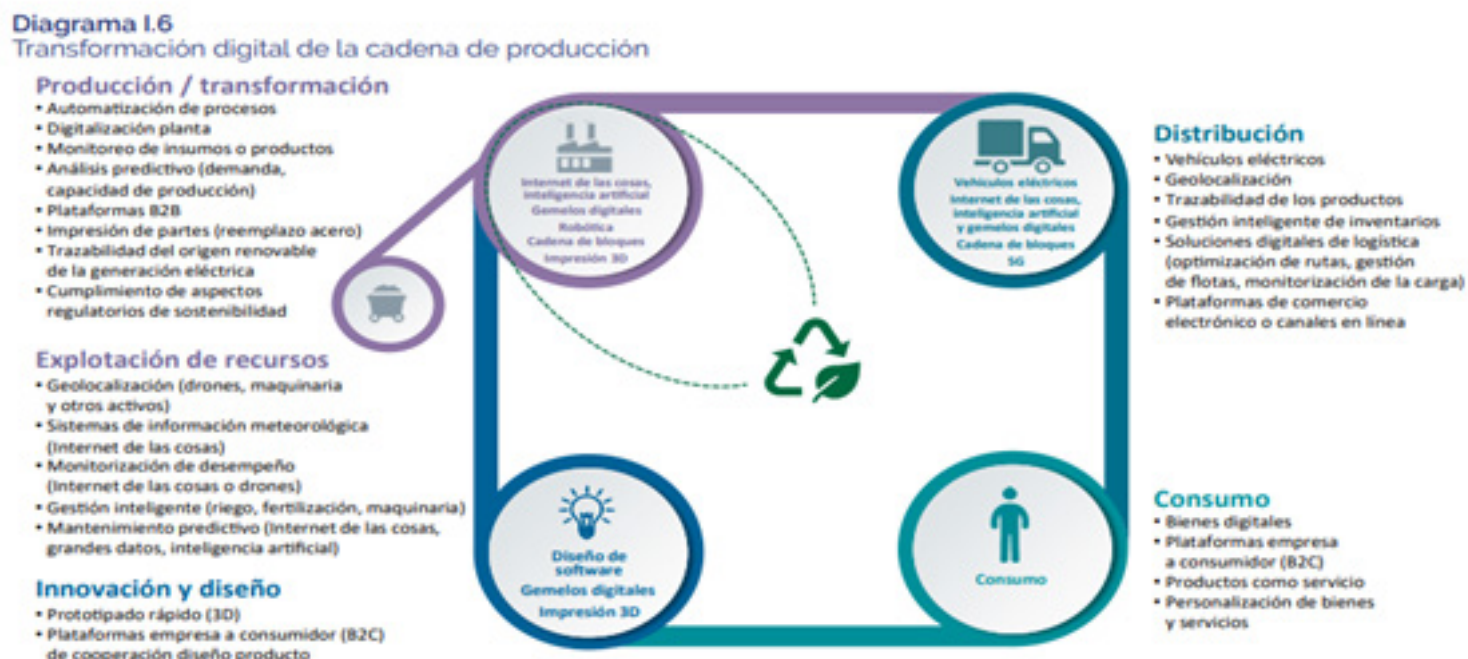

Gráfico 1. Transformación digital de la cadena de producción.

Fuente: (CEPAL, 2021)

En los distintos procesos, las TICS aportan en desarrollo y automatización, en palabras de (Secretaría de Estrategias Industriales, 2017) la fabricación avanzada supone la generación y aplicación de conocimiento, experiencia y tecnología de vanguardia para la creación de productos, componentes y servicios asociados de alto valor añadido Resaltando herramientas como:

- El Cloud Computing, ofertas de nube en ancho de banda para almacenamiento, aplicaciones y servicios, donde el valor de confidencialidad y la propiedad de los datos es destacada.

- El Big Data, tratamiento de información de grandes volúmenes de datos a alta velocidad, ayudando a tomar decisiones.

- Las redes sociales, permite el diálogo, la comunicación y el intercambio de información.

- Las impresoras 3D, sistema por aglomeración de polvo, metal o plástico que permite la fabricación rápida de piezas en pequeñas series y complejas.

- EL Internet de las cosas (IOT)
- Robots inteligentes y máquinas, capaces de interactuar de forma inteligente.

- La ciberseguridad y el aprovechamiento del cloud computing.

- Calidad de la conectividad, al interactuar en tiempo real el mundo físico con el virtual.

- Energía eficiente y descentralizada.

- Industrialización virtual.

- Factorías 4.0, desarrollando la realización de productos, utilizando sensores, impresoras 3D o robots inteligentes.

El término "Industria 4.0" supone la digitalización y conexión de todas las unidades productivas en una economía. En virtud de ahondar en el desarrollo de estas tecnologías y herramientas que representan un gran avance en las TIC, es importante referir la clasificación realizada por (CEPAL, 2021), de acuerdo a su aplicación en las cadenas productivas:

1. Analítica avanzada e inteligencia artificial: el uso de algoritmos y de máquinas de alto poder computacional permite la identificación de patrones y el análisis

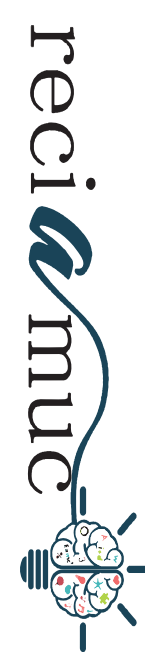




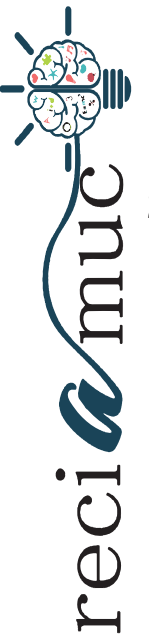

predictivo para facilitar el aprendizaje y la toma de decisiones de forma automatizada.

2. Internet de las cosas: el uso de sensores y actuadores permite utilizar dispositivos o productos inteligentes e interconectados, accesibles o rastreables en forma remota. Posibilita desarrollar "redes inteligentes" para automatizar o mejorar la efectividad de los procesos, particularmente cuando interactúa con tecnologías como la analítica avanzada.

3. Robótica avanzada: los avances en la inteligencia artificial, en la visión por computadora, en los sensores, en los motores y en la hidráulica permiten que los robots realicen tareas cada vez más complejas, con patrones menos repetitivos y predecibles, por lo que augura un avance en la robótica industrial para los años venideros.

4. Servicios en la nube y plataformas digitales: espacios en Internet que facilitan la ejecución de aplicaciones o programas en un mismo lugar para satisfacer distintas necesidades; reduciendo la necesidad de almacenamiento y de procesamiento en los computadores y los dispositivos locales, facilitando la transmisión de información.

5. Cadena de bloques: libro digital que trabaja con un registro único, consensuado y descentralizado para validar información y transacciones. El registro se distribuye en varios nodos de una red y en cada bloque se almacena una cantidad de registros o de transacciones válidas.

6. Navegación autónoma y semiautónoma: comprende los vehículos maniobrados sin intervención humana o con una intervención reducida. Incluye tanto automóviles, trenes y camiones como drones pilotados por un operador

7. Impresión 3D: pertenece a la familia de técnicas conocidas como manufactura aditiva. Los procesos aditivos permiten construir objetos mediante la creación y consolidación de capas, a diferencia de las técnicas de moldeo o sustractivas; así la impresión 3D tiene aún un importante potencial de aceleración tanto en términos de manufactura directa de productos y piezas como en la elaboración de herramientas y moldes.

8. Realidad virtual y realidad aumentada: el uso de tecnologías inmersivas como la realidad virtual y la realidad aumentada permite reinventar la forma de crear y de experimentar contenido. El uso de tecnologías inmersivas tiene múltiples impactos para los negocios, como la reducción de costos de producción mediante prototipos virtuales y la reducción de barreras a la entrada para nuevos creadores de contenido. (CEPAL, 2021)

Enmarcado en el desarrollo de estas herramientas tecnológicas, bastos autores han planteado que nos encontramos en una nueva revolución industrial, refiriendo a la aplicación de éstas a escala industrial, es decir, mediante sistemas automatizados en los procesos productivos y la interconexión de sus unidades.

Por su parte refiere que la (UNCTAD, 2021) Inteligencia Artificial (IA), por ejemplo, combinada con la robótica puede transformar los procesos productivos y comerciales; mientras que la impresión 3D permite una producción de bajo volumen más rápida y económica, así como la creación rápida e iterativa de prototipos de nuevos productos.

Es importante destacar como ya para 2018, estas 11 tecnologías representaban un mercado de 350.000 millones de dólares, tal como lo señala el informe de la (UNCTAD, 2021), quienes basados en estimaciones, pronosticaron desde entonces un crecimiento para 2025 que alcanzaría los 3,2 billones de dólares alrededor de todo el mundo, tal como se indica en el gráfico 2. 
2018

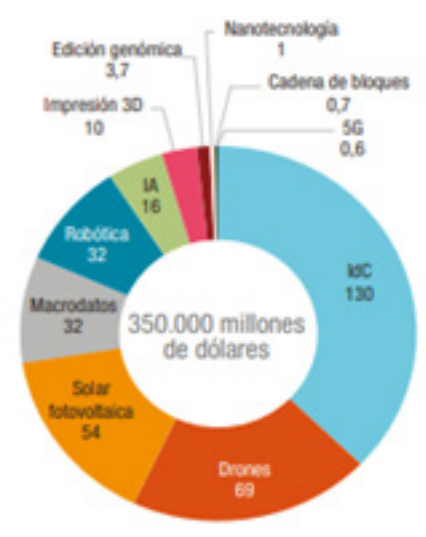

2025

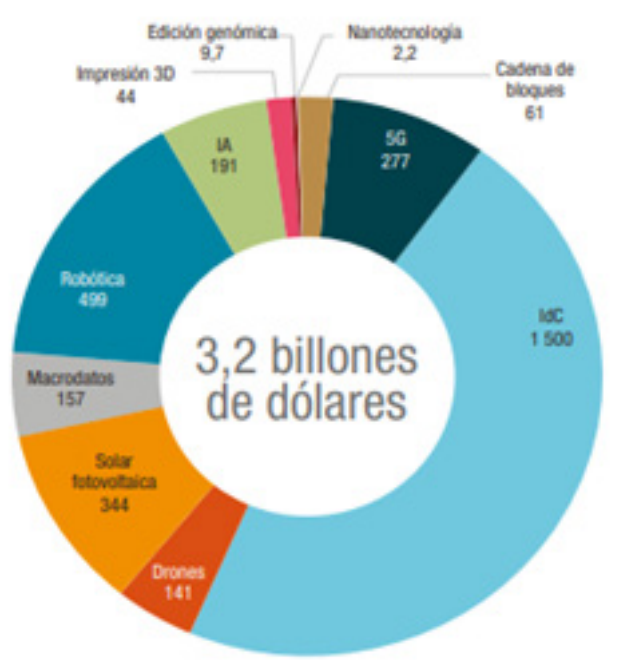

Gráfico 2. Estimaciones del tamaño del mercado de las TIC 2018 a 2025

(Expresado en miles de millones de dólares)

Fuente: (CEPAL, 2021)

\section{Contribucion de las Tics en la agroindus- tria alimentaria}

Es conocido la importancia que reviste el sector agroalimentario para la economía en el mundo no obstante sus productividad no es necesariamente la más exitosa, al menos no es en el caso de América latina, salvo excepciones su productividad es significativamente menor a la del promedio de la región y a la de los países más desarrollados (CEPAL, 2021)

En tal sentido la evolución de las tecnologías representa una oportunidad para impulsar no solo su productividad, sino la especialización en los procesos de logística y de distribución dentro del sector agrícola y el sector agroalimentario. En efecto, las tecnologías digitales pueden significar una ayuda sustancial a los agricultores para poder producir eficientemente y en el marco de procesos que representen soluciones sostenibles ante el cambio climático.

Un ejemplo de esta contribución señala (CEPAL, 2021), es la incorporación de sensores a las máquinas agrícolas convencionales, como los tractores, las pulverizadoras y las cosechadoras, los cuales pueden permitirle transformarlas en redes de dispositivos inteligentes para monitorear su rendimiento, "piloto automático o sensores para la distribución de semillas y la pulverización".

Por su parte, la aplicación de la " analítica avanzada" resulta útil a dicho sector, dado que proporciona el procesamiento de la información generada con sensores, satélites, GPS en virtud de tomar decisiones oportunas que optimice el proceso productivo.

Otra contribución que se puede señalar es la utilización de automóviles autónomos o semiautónomos para disminuir costos y consumo de energía, con altos índices de seguridad y precisión.

En síntesis las tics representan una potencial oportunidad en todos los procesos de la cadena de valor, dentro del sector agroalimentario, desde sus inicios hasta el destino final del producto. En tal sentido, cabe destacar el señalamiento de (CEPAL, 2021) de algunas de estas contribuciones por cada uno de los procesos que componen la cadena de valor, de la siguiente forma: 


\section{Compras}

a. Suministro de insumos y equipos para todo el proceso

b. Siembra, riego, fertilizantes y control de plagas

c. Recolección de productos, transporte a plantas y almacenamiento

d. Conversión en productos finales

e. Logística hasta destino y comercialización de productos

f. Plataformas de comercio digital para adquisición de insumos, herramientas, maquinaria agrícola y vehículos especializados (por ejemplo, Agrofy y Agroads)

II. Cultivo

a. Gestión inteligente del riego a través de sistemas de Internet de las cosas

b. Plataformas de seguimiento de cultivo para facilitar la toma de decisiones a través de sistemas de información geográfica en la nube

c. Robots autónomos para inspección automatizada (por ejemplo, Mineral, de Alphabet)

d. Monitoreo del cultivo mediante drones para construcción de mapas de humedad, plagas, geolocalización de elementos y otros

III. Recolección y transporte

a. Automatización de la elección del momento de cosecha a través de sensores y analítica avanzada

b. Gestión inteligente de inventarios y activos a través de tecnologías como sistemas de identificación por radiofrecuencia

c. Optimización de rutas y gestión de flotas a través de herramientas de analítica avanzada

IV. Transformación: a. Automatización de procesos de producción en fábrica a través de robots industriales

b. Mantenimiento predictivo de maquinaria y equipamiento de producción a través de uso de sensores

\section{Distribución}

a. Plataformas de comercio digital para facilitar la comercialización y la venta de productos finales

b. Herramientas de marketing digital para anunciar y promover los productos (por ejemplo, Agroads)

c. Utilización de sensores para monitorear cadena en frío .(CEPAL, 2021)

Desde la perspectiva de Domene, (2019) la aplicación de las TICS a la industria en la agroindustria alimentaria se enfoca en trazabilidad, gestión, logística y control. Plantea la incorporación de técnicas y sistemas de vigilancia tecnológica, mediante las herramientas de simulación, se basa principalmente en el impacto que ha tenido la tecnología en las demandas del consumidor, enfocadas cada día más en la seguridad del producto final; de hecho, supone que tener y proveer de accesibilidad a la información, significará una ventaja competitiva en este sector.

Respecto a la trazabilidad señala de igual forma, la importancia que reviste actualmente, las ventajas de aquellos sistemas que le permiten al consumidor conocer quién, cómo, dónde y cuándo se ha producido el alimento que va a consumir.

En torno a la cadena de distribución, las TICS proporcionan la posibilidad de hacerlas más corta mediante el uso de herramientas que digitalicen los procesos, creando en "cercanía digital", eliminando tiempos y esfuerzos burocráticos en la relación cliente-proveedor, con elementos de control que faciliten la anticipación. (Domene, 2019) 


\section{Las Tic en el desarrollo sostenible de la industria agroalimentaria en america latina}

El desarrollo e impulso de las tics en las distintas regiones para 2018 desde los sectores económicos ya avizoraba un vertiginoso crecimiento, la CAF indicaba el desarrollo del ecosistema digital de acuerdo al índice de desarrollo del ecosistema digital, que puede observarse en el Gráfico 3:

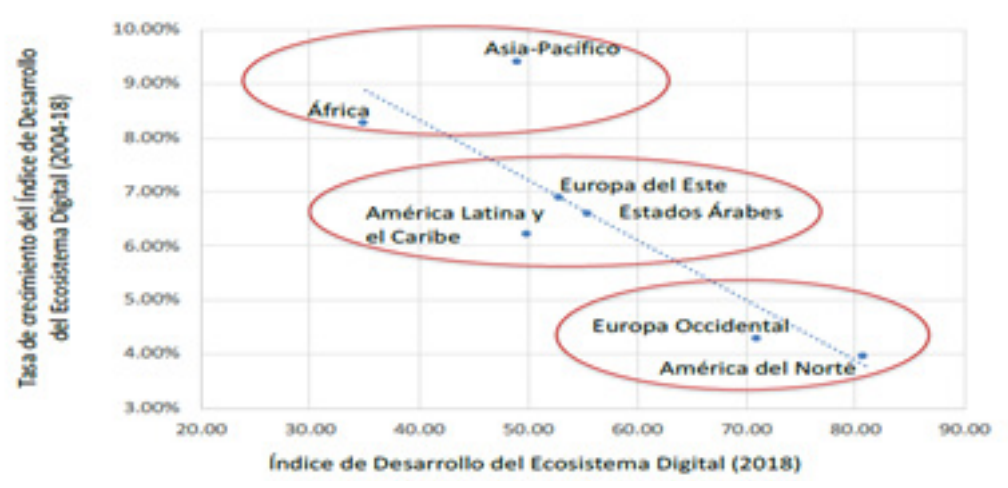

Gráfico 3. Índice de Desarrollo del Ecosistema Digital (2018) vs. Tasa de Crecimiento (2004-18)

Fuente: Observatorio CAF del ecosistema digital, 2020.

De tal forma, que se puede referir que América Latina se encuentra en una situación de ventaja relativa, para aprovechar todo el potencial que las tics pueden ofrecer, pero con menores tasas de crecimiento que el resto de las regiones. Esto es un asunto importante que cada país del mundo debe atender, conjuntamente con el apoyo de las organizaciones internacionales que buscan la equidad tecnológica, sobretodo en estos momentos donde la crisis a causa de la pandemia de la COVID-19, ha obligado que el uso de las TICs sea imprescindible para el desarrollo de prácticamente cualquier actividad organizacional y personal (CEPAL, 2021).

De cualquier forma, es importante resaltar que el avance tecnológico para la economía es indetenible, la (UNCTAD, 2021) señala al respecto, que en la actualidad, el mundo está llegando al final de la fase de implantación de la "Era de las TIC" e inicia la fase de instalación de un nuevo paradigma, que incluye tecnologías de frontera, revolución a veces denominada cuarta revolución industrial o Industria 4.0 (gráfico 4).

Más allá de adentrarse en este debate, es menester precisar por una parte, que las TICS han tenido un crecimiento acelerado, y las potencialidades que estas herramientas tecnológicas usadas representan para el sector agroalimentario, sobre todo para los agricultores, a quienes se les exige mayor armonía en sus procesos productivos con el medio ambiente propiciando el desarrollo sostenible, ante las problemática del cambio climático. 


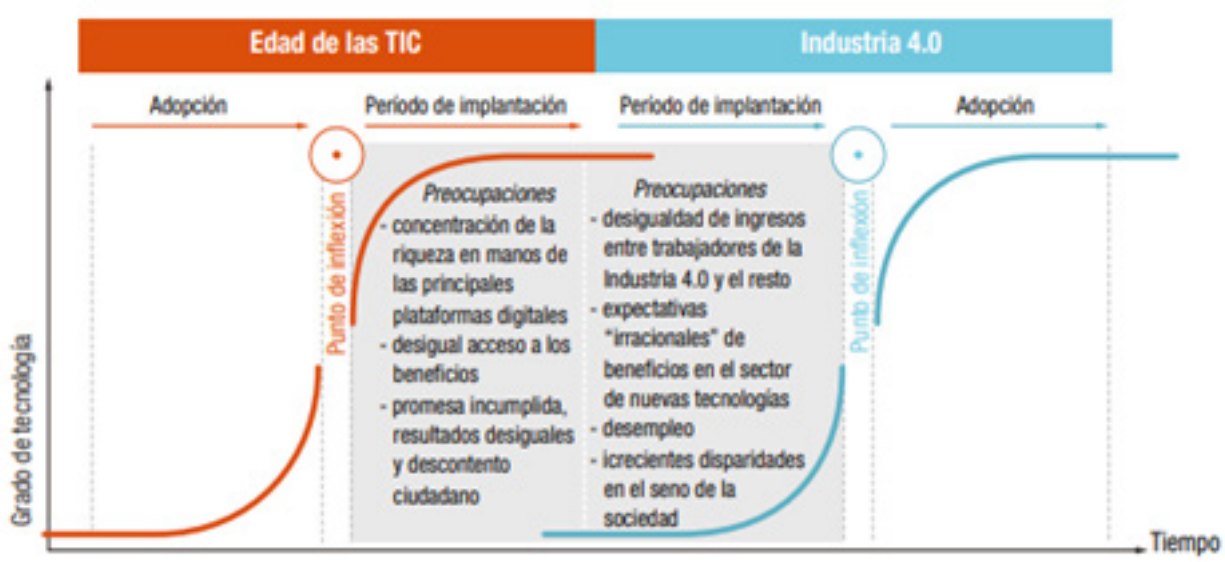

Gráfico 4. Evolución de las tecnologías y su relación con las desigualdades.

Fuente: (UNCTAD, 2021)

Ciertamente, advierte la (UNCTAD, 2021) ciertas desigualdades entre países ante el acceso de estas tecnologías, destacando una relación directa entre la revolución tecnológica y las desigualdades, por lo cual, se puede destacar la importancia que reviste que su aplicación debe ser en marco del desarrollo sostenible.

Así se puede constatar que el desarrollo de la economía digital está en pleno proceso evolutivo, y ante esto el estado y las empresas deben adaptar sus sistemas y modelos de gestión, hacia esta transformación dinámica y compleja, desafío que supone la digitalización, tomando en cuenta el desarrollo sostenible, que implica, en un sentido, mas ético, ecológico y de calidad, bajo la premisa de las tres dimensiones del desarrollo sostenible: crecimiento, igualdad y sostenibilidad.

En este aspecto se puede afirmar que la evolución hacia la economía digitalizada permitiría no solo satisfacer a los consumidores con productos inteligentes, y en consecuencia beneficiando al mismo, sino que, estas nuevas formas de consumo actualmente se asocian con beneficios potenciales derivados de la reducción de la materialización y de decisiones medioambientales más sostenibles, en la medida que se basen en más y mejor información (por ejemplo, la relacionada con la huella ambiental de un producto) o recompensen prácticas más inocuas con el medioambiente. (CEPAL, 2021)

Cabe acotar, que esta transformacion económica se desarrolla en el marco de la búsqueda de soluciones por parte de las empresas, en cuanto al impacto ambiental en sus modos de producción y de distribución, en tal sentido, la digitalización de la economía proporciona soluciones viables , los nuevos modelos de producción inteligente, pueden aumentar la competencia incluso con menor impacto ambiental, y las herramientas tecnológicas ya esbozadas, permiten nuevas alternativas a las organizaciones para modificar y disminuir el impacto que puedan estar teniendo en el cambio climático.

En todo caso, es necesaria un sentido ético y sostenible de gobernanza y su forma de adoptar estas tecnologías en pro del crecimiento y la igualdad. "Aunque la digitalización puede contribuir en gran medida en las tres dimensiones del desarrollo sostenible (crecimiento, igualdad y sostenibilidad), su impacto neto dependerá de su grado de adopción y de su sistema de gobernanza" . (CEPAL, 2021)

La Pandemia Covid 19 y en consecuencia el cierre de empresas y actualmente su reapertura, ha significado que estos cambios 
en la digitalización de los procesos económicos se hayan dado de forma abrupta y acelerada, ya que la utilización de las tecnologías digitales como respuesta a esa situación, ha conllevado a una relación más directa y permanente con las TICS, e incluso su adopción se ha convetido en prioridad para la dinamización del aparato productivo, convirtiendo este nuevo modo de producción y de consumo en irreversible ; por lo tanto, es necesario disminuir las brechas tecnológicas y facilitar el libre acceso, esto será garantía de la sostenibilidad.

A continuación se esboza grafico 5, que refiere de acuerdo a la Agenda 2030 para el Desarrollo Sostenible y el cumplimiento de los Objetivos de Desarrollo Sostenible (ODS) de la CEPAL, (2021), las prioridades que debe asumir la economía digital en cada uno de los sectores hacia la consecución de estos objetivos. Se puede precisar con mayor énfasis el sector agroalimentario, que no escapa de esta realidad, y por ser prioritario, requiere aún más esfuerzo para el logro de estos fines.

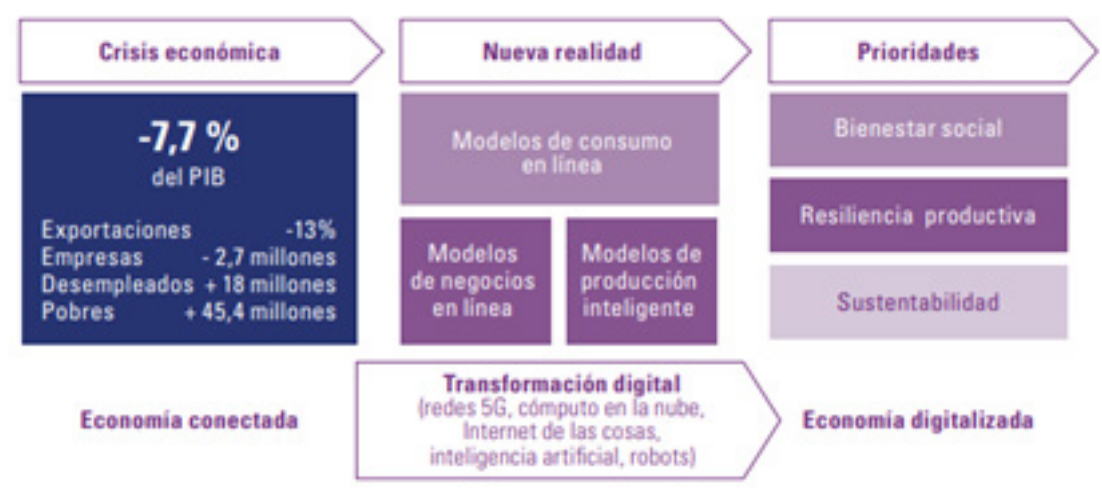

Gráfico 5. Reactivación para América Latina y el Caribe

Fuente: (CEPAL, 2021)

\section{Contribuciones de las TICS en el desarro- Ilo sostenible}

Se pueden indicar algunas contribuciones en específico que proporcionan las TIC en esta nueva fase de la digitalización económica, al desarrollo sostenible:

- Las innovaciones tecnológicas que pueden ser realizadas mediantes las nuevas tecnologías, genera un menor impacto ambiental, ya que distintas herramientas como los simuladores, y otros, permiten la optimizar el uso de los recursos; de hecho, a medida que estas tecnologías evolucionen y converjan con la biotecnología y la nanotecnología, podrían generar innovaciones exponenciales que coadyuvarían a un futuro sostenible.

- Facilita la "desmaterialización" económica, esto es mediante la nueva oferta de servicios y productos digitales, que no requieren de emisiones de carbono para su producción, así como el hecho de que evita desplazamientos innecesarios, y posibilita la exportación de estos bienes y servicios digitales.

- De forma general se puede afirmar que estas tecnologías como el Internet de las cosas y la inteligencia artificial contribuyen notablemente a disminuir las emisiones globales de carbono, la Cepal (2021), estima que hasta en un 15\%, lo que significaría un tercio de la reducción del $50 \%$ propuesta para 2030 . Todo esto puede suponer la compensación de los posibles efectos negativos de la aplicación de estas tecnologías, que implican un gran consumo de energía y una masiva generación de desechos electrónicos (e-waste) y la explotación de recursos

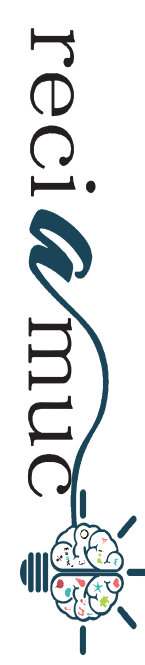


naturales, como el cobre y el litio (Malmodin \& Lunden, 2018)

Algunas de las limitaciones que deben mencionarse al respecto del desarrollo de las tecnologías es que éstas requieren de altos consumos de energía (centros de datos y redes), procesos de producción contaminantes de hardware (pantallas), y modelos de negocios que incentivan el recambio de dispositivos en períodos cortos.

Sin embargo, los indicadores de intensidad muestran que en el consumo de energía y la generación de huella de carbono se han logrado importantes avances, ya que cada suscripción o cada GB transmitido tiene cada vez un menor impacto (81 kg CO2e/suscripción en 2015 frente a 134 kg CO2e/suscripción en 2007, y 0,8 kg CO2e /GB en 2015 frente a 7 en 2007) (Malmodin \& Lunden, 2018)
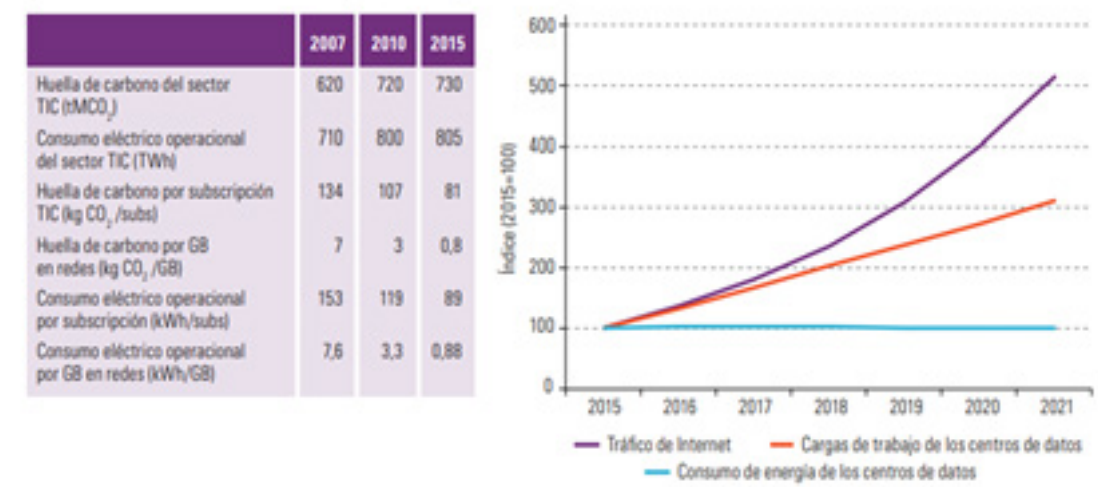

Gráfico 6. Huella de Carbono y de energía de las tecnologías de TIC

Fuente: (Malmodin \& Lunden, 2018)

\section{Conclusión}

El desarrollo de las tecnologías de TIC ha sido vertiginoso y sigue su auge en este mundo globalizado, por lo que no hay ninguna actividad económica que esté exenta de su utilización. El sector agroalimentario es uno de los más importantes para la sociedad, y poder suministrar la alimentación ahora cuando el distanciamiento social, obliga a que la aplicación de estas, en todas las etapas de la producción para obtener resultados exitosos.

El actual escenario pandémico y en el marco los impactos ambientales que han generado el cambio climático, esto ha retado el rol del agricultor, a producir de forma eficiente. Las TICS constituyen hoy en día una alternativa viable para disminuir estos impactos, y convertirse en la nueva vía, con un correcto y eficaz uso.
Aun y cuando esto pueda traer algunos efectos, el avance tecnológico es irreversible, y es un desafío para el sector agroalimentario, asumir esta tendencia en todos los eslabones de la cadena de producción supone cuidar los efectos resultante de la digitalización de la economía, tales como, el desplazamiento de la fuerza labor, o un considerable potencial de cambio en las condiciones del trabajo, y es que el cambio tecnológico afecta a las desigualdades a través de sus repercusiones en el empleo, los salarios y los beneficios. Por otro lado se encuentra el hecho que mayor desarrollo digital genera efectos negativos asociados al consumo de energía.

A pesar de esto, las TIC siguen representando mayores ventajas, ya que la prestación de servicios y producción de bienes de forma digital disminuye las emisiones de carbono. 
De tal forma, que la digitalización debe tomar en consideración así advertencias esgrimidas por la Naciones Unidas, en torno a las naciones menos favorecida, como América Latina y las desigualdades que significa el acceso a estas nuevas tecnologías en igualdad de condiciones.

Es por esto que se hace imperativo el desarrollo de documentos teóricos orientadores el apoyo estatal, y de empresas privadas institutos tecnológicos y en fin la iniciativa del propio sector agrícola en el desarrollo e inversión en las nuevas tecnologías, para no solo ponerse a la lid de los avances y el vorágine tencologico actual, sino estar en la capacidad de anticiparse a la nuevos tiempos de economía digital que esta en la palestra mundial.

Si bien es cierto que los avances tecnológicos son esenciales para el desarrollo sostenible, también lo es que pueden propiciar el enquistamiento de las desigualdades o la aparición de otras nuevas, de allí que el rol de las autoridades públicas y privadas es maximizar las ventajas potenciales y atenuar los resultados perjudiciales, garantizando el acceso universal. Un requisito importante es una gobernanza nacional que sea eficaz; el Estado debe establecer la visión, la misión y el plan para crear y configurar un mercado de innovaciones inclusivas y sostenibles.

De igual forma, para que se cumpla el Desarrollo Sostenible es menester que las la sociedad sea garante mediante actos de seguimiento sobre las desigualdades, basados en medidas reguladoras e instrumentos económicos y fiscales. También pueden garantizar que los grupos vulnerables y de pocos recursos tengan acceso a nuevos y valiosos bienes y servicios, algunos de los cuales pueden ser subvencionados $u$ ofrecidos gratuitamente.

En conclusión La penetración de la informática en los procesos industriales lleva observándose desde la década de los años setenta del pasado siglo y es a partir del inicio de la década de los años 2000 cuando se está intensificando la aplicación de innovaciones tecnológicas digitales en las actividades industriales, y en específico en el sector agroalimentario, que permite generar una cadena de valor más ágil y eficiente. Avanzar en las tecnologías digitales deben utilizarse para construir un nuevo futuro mediante el crecimiento económico, la generación de empleo, la reducción de la desigualdad y una mayor sostenibilidad.

\section{Bibliografía}

Calandra, P., \& Araya, M. (2009). Conociendo las TIC . Chile: Marco Mocelli Inestrosa.

Camara Valencia. (22 de 10 de 2021). Tecnologìa para los negocios. Obtenido de https://ticnegocios.camaravalencia.com/servicios/tendencias/ como-usaran-las-tic-las-empresas-lideres-en-alimentacion-en-los-proximos-anos/

Cedeño Troya, F. (2018). Influencia de la inversión en tecnologías de la información como factor de competitividad de las empresas Pymes. Revista Espacios, 39(32), 23.

CEPAL. (2021). Tecnologías de la información y las comunicaciones (TIC). Recuperado el 31 de Mayo de 2021, de https://www.cepal.org/es/temas/tecnologias-de-la-informacion-y-las-comunicaciones-tic

CEPAL. (2021). Tecnologías digitales para un nuevo futuro. Santiago: Naciones Unidas.

Cobo Romaní, J. (2009). El concepto de tecnologías de la información. Benchmarking sobre las definiciones de las TIC en la sociedad del conocimiento. Revista Zer, 14(27), 295 - 318.

Condori Cruz, R. (2020). Impacto y desafios de las tecnologías de la información y comunicacines en tiempos de pandemia COVID - 19. Revista Científica Investigación Andina, 20(2).

Da Silva, F., De Furquim, J., \& Núñez, G. (2020). La libre concurrencia en la economía digital: las micro, pequeñas y medianas empresas (mipymes) en América Latina y el impacto del COVID-19 (Publicación de las Naciones Unidas ed.). Naciones Unidas: CEPAL.

de la Fuente, A. (Septiembre de 2008). Inversión en TICs y productividad: Fundación de estudios de economía aplicada Un breve panorama y una primera aproximación al caso de las regiones españolas. 
Domene, A. (04 de Abril de 2019). Agrotansformació Digital. Obtenido de El Virus que afecta la eficiencia de los Modelos de Gestiòn Agroalimentarios: https://antoniodomene.com/modelos-de-gestion-agroalimentarios/

Espinal, A. (12 de Septiembre de 2019). El papel de las TICs en las empresas. Revista Tecnológica Educativa, 1(1), 13-14.

Hernández, A. (Mayo31 de 2021). Economiatic. Recuperado el 2021, de Concepto de TIC: Tecnologías de la Información y la Comunicación: https:// economiatic.com/concepto-de-tic/

Instituto Nacional de Estadística y Censos del Ecuador. (2015). Empresas y TIC (Tecnologías de la Información y la Comunicación). INEC. Ecuador: INEC.

Instituto Nacional de Estadistica y Censos. (Julio, 2011). Censo Nacional Económico. Informe Oficial INEC, INEC, Ecuador.

Instituto Nacional de Estadpistica y Censos. (Julio, 2011). Censo Nacional Económico. Informe Oficial INEC, INEC, Ecuador.

ITU. (2021). International Telecommunication Union (ITU). Obtenido de Measuring digital development: Facts and figures 2020: https://www.itu.int/ en/ITU-D/Statistics/Pages/facts/default.aspx

Ledesma Silva, Y., \& Cobos Reina, A. (Marzo de 2018). La Triple H 凶elice de las TIC en Ecuador. JOURNAL OF SCIENCE AND RESEARCH: REVISTA CIENCIA E INVESTIGACIÓN, 2(9), 41-48.

Malmodin, J., \& Lunden, D. (2018). The Energy and Carbon Footprint of the Global ICT and E\&M Sectors 2010-2015. Sustainability , 10(9), 3027.

Monroy, G. (14 de Junio de 2020). GICES. Recuperado el 31 de Mayo de 2020, de Herramientas Tecnológicas Aplicadas a la Educación a Distancia: https://www. gicesperu.org/articulo. php?id=q+sNp2eAe7ON4EYpqsM
Observatorio CAF del Ecosistema Digital. (Abril 2020). El estado de la digitalización de América Latina frente a la pandemia del COVID-19. Informe Oficial, Corporación Andina de Fomento.

Ochoa Guevara, S., Medina Acero, C., Santamaría Burgos, L., Ramírez Campos, D., Paramo Renza, E., \& Ochoa Guevara, N. (Diciembre de 2020). Reto de las mipymes para. RHS. Revista. Humanismo y Sociedad incursionar en el marketing digital en tiempos de COVID-19, 8(2), 132-145.

Peirano, F., \& Suárez , D. (2006). TICS Y EMPRESAS: PROPUESTAS CONCEPTUALES PARA LA GENERACIÓN DE INDICADORES PARA LA SOCIEDAD DE LA INFORMACIÓN. Revista de Gestão da Tecnologia e Sistemas de Informação, 3(2), 123-142.

Quispe Otacoma, A., Padilla Martínez, M., Telot González, J., \& Nogueira Rivera, D. (Enero-Abril de 2017). Tecnologías de información y comunicación en la gestión empresarial de pymes comerciales. Revista Ingeniería Industrial, XXXVIII(1), 81-92.

Rodríguez, K., Ortiz, O., Quiroz, A., \& Parrales, M. (5 de Noviembre de 2020). El e-commerce y las Mipymes en tiempos de Covid-19. Revista Espacios, 41(42), Art. 9.

Secretaría de Estrategias Industriales. (2017). La Digitalización y la Industria 4.0. Madrid: Industria CCOO.

Tricoci, A., Corral, P., \& Rosenthal, A. (Enero-Junio de 2016). Inversiones en Tecnologías de La Información y Comunicaciones y su Impacto en la Competitividad. Revista Científica Visión de Futuro, 20(1), 87-104.

UNAN. (2021). La transformación digital durante la pandemia de la COVID-19 y los efectos sobre la docencia. Foro. Nicaragua: Universidad Nacional Autónoma de Nicaragua (UNAN).

UNCTAD. (2021). INFORME SOBRE TECNOLOGIA E INFORMACIÒN 2021. Ginebra: Naciones Unidas.

UNESCO. (2021). Las TIC en la educación. Obtenido de https://es.unesco.org/themes/tic-educacion

\section{CITAR ESTE ARTICULO:}

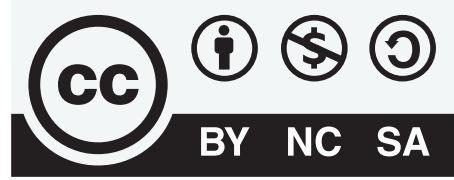

Ordoñez García, J. E., Merizalde Andrade, C. E., \& Villamar Piguave, W. G. (2021). TIC y su contribución para el desarrollo sostenible en la agroindustria alimentaria. RECIAMUC, 5(4), 22-36. https://doi.org/10.47464/reciamuc/5.(4). noviembre.2021.22-36 